\title{
Adesão à alimentação escolar por adolescentes brasileiros: determinantes individuais e do contexto escolar
}

\author{
Acceptance of school food by Brazilian adolescents: \\ individual and school context determinants
}

Diôgo Vale (https://orcid.org/0000-0003-2636-4956) 1,3

Clélia de Oliveira Lyra (https://orcid.org/0000-0002-1474-3812) ${ }^{2}$

Thais Teixeira dos Santos (https://orcid.org/0000-0002-2568-2007) ${ }^{3}$

Camila Valdejane Silva de Souza (https://orcid.org/0000-0001-7146-3406) ${ }^{1}$

Angelo Giuseppe Roncalli (https://orcid.org/0000-0001-5311-697X) ${ }^{4}$

${ }^{1}$ Programa de Pós-

Graduação em Saúde

Coletiva, Universidade Federal do Rio Grande do Norte (UFRN). BR-406, Km 145, Bairro Planalto. 59570-000 Ceará-Mirim RN Brasil.diogo.vale@ escolar.ifrn.edu.br ${ }^{2}$ Departamento de Nutrição, UFRN. Natal RN Brasil. ${ }^{3}$ Instituto Federal de Educação, Ciência e Tecnologia do Rio Grande do Norte. Mossoró RN Brasil.

${ }^{4}$ Departamento de Odontologia, UFRN. Natal RN Brasil.

\begin{abstract}
The scope of this study was to evaluate the acceptance of school food by Brazilian adolescents, taking individual and school context determinants into consideration. A total of 10,262 adolescents in public schools of the 2015 Brazilian National Survey of School Health (PeNSE 2015) were assessed. Multilevel regression was used to estimate the effect of variables of the individual context and the school environment on low acceptance of school food by adolescents. The prevalence of low acceptance (consumption of school meals $\leq 2$ days/week) was $64.2 \%$ in this group. The explanatory model showed less acceptance of this practice among female adolescents $(P R=1.09$; CI: 1.03-1.14), among those aged 15 years or older $(P R=1.08$; CI: 1.02-1.14), those who regularly consumed soft drinks ( $P R=1.09$; CI: 1.03-1.15), those who did not regularly have lunch and dinner with parents and guardians $(P R=1.08$; $C I$ : 1.02-1.14), and those who lived in households with less than 4 people ( $P R=1.07 ; C I$ : 1.02-1.13). Concerning the school environment, studying in schools with more than 1,000 students $(P R=1.22$; $C I$ : 1.12-1.33) and the presence of a cafeteria $(P R=1.15 ; C I: 1.08-1.22)$ led to low acceptance. The results revealed the importance of the school context in determining the consumption of school meals by adolescents.
\end{abstract}

Key words School food, Food consumption, Eating habits, Adolescent
Resumo Objetivou-se avaliar a adesão à alimentação escolar por adolescentes brasileiros considerando determinantes individuais e do contexto escolar. Foram avaliados 10.262 adolescentes de escolas públicas da Pesquisa Nacional de Saúde do Escolar de 2015. Regressão multinível foi utilizada para estimar o efeito de variáveis do contexto individual e do ambiente escolar na ocorrência da baixa adesão à alimentação escolar por adolescentes. A prevalência dessa baixa adesão (consumo da alimentação escolar $\leq 2$ dias/semana) foi $64,2 \%$ nesse grupo. $O$ modelo explicativo evidenciou menor adesão a essa prática entre adolescentes do sexo feminino $(R P=1,09 ; I C: 1,03-1,14)$, entre aqueles com 15 anos ou mais de idade $(R P=1,08$; IC: 1,02-1,14), que tinham consumo regular de refrigerantes $(R P=1,09 ; I C: 1,03-1,15)$, que não realizavam regularmente almoço e jantar com pais e responsáveis $(R P=1,08 ;$ IC: $1,02-1,14)$, e que moravam em domicílios com menos de 4 pessoas ( $R P=1,07$; IC: 1,02-1,13). Em relação ao ambiente escolar, estudar em escolas com mais de 1.000 alunos ( $R P=1,22$; IC: 1,12-1,33) e com presença de cantina $(R P=1,15$; IC: $1,08-1,22)$ determinaram a baixa adesão. Os resultados demonstram importante efeito do contexto escolar na determinação do consumo da alimentação escolar por adolescentes.

Palavras-chave Alimentação Escolar, Consumo de Alimentos, Hábitos Alimentares, Adolescente 


\section{Introdução}

O Programa Nacional de Alimentação Escolar (PNAE), com cobertura universal para estudantes matriculados em escolas públicas do Brasil, é uma ação norteada pela Política Nacional de Segurança Alimentar e Nutricional ${ }^{1}$ e pela Política Nacional de Alimentação e Nutriçãó ${ }^{2}$. Esse programa vinculado às redes de educação municipais, estaduais e federais tem como objetivos principais a oferta de refeições adequadas e saudáveis, além da educação alimentar e nutricional $^{3}$. Por isso, trata-se de uma importante estratégia para melhoria dos padrões alimentares das crianças e dos adolescentes brasileiros.

Um dos desafios do PNAE é a adesão dos estudantes às refeições saudáveis ofertadas, principalmente, por parte dos adolescentes, os quais apresentam maior tendência em consumir regularmente alimentos ultraprocessados ${ }^{4,5}$. Todavia, esses alimentos são restritos nas normativas do Programa com o objetivo de promover alimentação adequada e saudável no ambiente escolar³.

Apesar de o consumo regular das refeições ofertadas pelas escolas ter aumentado entre 2012 $(22,8 \%)^{6}$ e $2015(38,1 \%)^{7}$ entre adolescentes brasileiros, conforme apontado por resultados da Pesquisa Nacional de Saúde do Escolar (PeNSE), prevalece a baixa adesão ao $\mathrm{PNAE}^{8}$ por parte desse grupo etário. Esses resultados estão muito aquém da cobertura universal preconizada pelo Programa ${ }^{3}$.

Nesse contexto, a identificação dos determinantes da baixa adesão à alimentação escolar por adolescentes é necessária ao planejamento contínuo das ações no âmbito do PNAE. Estudos sobre essa questão já evidenciaram fatores individuais relacionados à melhor adesão às refeições ofertadas nas escolas públicas por adolescentes brasileiros, como suas características sociodemográficas - alunos do sexo masculino, pardos, que não moravam em capitais, que trabalhavam e cujas mães apresentavam baixa escolaridade; e uma rotina alimentar marcada por consumir café da manhã e almoçar com os pais regularmente ${ }^{9}$.

Além disso, o consumo satisfatório da alimentação escolar entre adolescentes foi associado positivamente ao consumo moderado e regular de feijão, vegetais crus ou cozidos, legumes cozidos e frutas ${ }^{10}$; e ao menor consumo regular de refrigerantes, salgadinhos de pacote, salgados fritos e guloseimas ${ }^{11}$. Apontando o potencial do PNAE no processo de melhoria dos padrões alimentares dos adolescentes brasileiros.

Verifica-se que a adesão à alimentação escolar foi avaliada a partir de determinantes individuais, relativos a comportamentos e contexto familiar. Porém, acredita-se que devem ser investigadas características do contexto escolar, as quais podem complementar o entendimento da rede de determinantes da adesão de adolescentes brasileiros às refeições ofertadas pelo PNAE. Tal hipótese baseia-se em resultados que indicaram associações entre o pior perfil de alimentos comercializados no ambiente escolar e as práticas alimentares de risco nutricional nessa faixa etária ${ }^{12,13}$.

Resultados do Estudo de Risco Cardiovascular em Adolescentes identificaram pior ambiente alimentar em escolas particulares, as quais apresentaram: maior venda de alimentos no interior das escolas, mais propagandas de alimentos ultraprocessados, presença de máquinas de venda automática de produtos industrializados, bem como vendedores ambulantes nas proximidades da escola. Entretanto, observa-se que as escolas públicas também possuem ambientes com disponibilidade de alimentos ultraprocessados, podendo ser um determinante do consumo irregular da alimentação escolar ${ }^{14}$.

Esse contexto das escolas, favorável ao consumo desse grupo de alimentos com alta densidade energética e grande quantidade de aditivos sintéticos, somado às dificuldades na execução do PNAE, podem ser considerados fatores associados à baixa adesão às refeições servidas entre esse grupo etário. Portanto, o objetivo deste trabalho foi avaliar a adesão à alimentação escolar por adolescentes brasileiros considerando determinantes individuais e do contexto escolar.

\section{Metodologia}

\section{Desenho do estudo e fonte de dados}

Foi realizado um estudo do tipo inquérito populacional utilizando o banco de dados dos níveis individuais e da escola de adolescentes brasileiros provenientes da "Amostra 2" da PeNSE 2015. Esse estudo foi conduzido pelo Instituto Brasileiro de Geografia e Estatística (IBGE) e pelo Ministério da Saúde, com apoio do Ministério da Educação. A PeNSE 2015 foi aprovada pela Comissão Nacional de Ética em Pesquisa (Conep), em 30 de março de $2015^{7}$, e o presente trabalho atende às questões de ética em pesquisa dispostas na Resolução no 510, de 07 de abril de 2016, do Conselho Nacional de Saúde ${ }^{15}$.

A “Amostra 2" da PeNSE 2015 totalizou 16.608 estudantes respondentes, distribuídos do $6^{\circ}$ ao $9^{\circ}$ ano do Ensino Fundamental, e da $1^{\text {a }}$ a $3^{\text {a }}$ série do Ensino Médio dos turnos matutino, 
vespertino e noturno. Estes estavam devidamente matriculados e frequentando regularmente escolas públicas e privadas situadas nas zonas urbanas e rurais de todo Brasil. Essa amostra permitiu a coleta de dados passíveis de comparação com indicadores nacionais e internacionais para o mesmo público, diferente do recorte da PeNSE 2015 chamado "Amostra 1", com representatividade apenas para alunos do $9^{\circ}$ ano do Ensino Fundamental ${ }^{7}$.

Essa pesquisa utilizou um plano de amostragem complexa, a fim de possibilitar a estimativa de parâmetros populacionais para cada uma das cinco macrorregiões do país (Norte, Nordeste, Sudeste, Sul e Centro-Oeste) e, consequentemente, para o Brasil. Compuseram a unidade informante todos os escolares das turmas selecionadas para a amostra e o responsável administrativo, tendo eles respondido a questões individuais e sobre a escola, respectivamente. Com isso, a unidade de análise total foi composta por 371 escolas, 653 turmas e 16.608 escolares selecionados, que concordaram em participar da pesquisa e informaram sexo e idade. A coleta dos dados individuais foi realizada em questionário eletrônico autoaplicável; e a coleta de dados da escola, com questionário aplicado ao responsável. Ambas aconteceram entre abril e setembro de $2015^{7}$.

A maior parte das variáveis do questionário da PeNSE 2015 apresentou percentual de não resposta inferior a $1,0 \%$. Os pesos amostrais associados a cada aluno participante da pesquisa, que teve seu questionário considerado válido pela equipe da PeNSE 2015, foram calculados após a coleta dos dados. Outros detalhes sobre o desenho amostral e informações sobre a pesquisa podem ser encontrados na publicação da PeNSE $2015^{7}$.

As análises deste estudo consideraram apenas 10.262 alunos presentes no banco de dados imputados original, que frequentavam 281 escolas públicas e dispunham de alimentação escolar. Para chegar a esse recorte do banco de dados, foram excluídos do banco inicial: (1º) 5.440 alunos de escolas privadas e (2o) 854 estudantes, cuja escola relatou não oferecer comida ou que não tinham dados disponíveis para essa questão.

\section{Variáveis do estudo}

As variáveis utilizadas no presente estudo foram coletadas nos seguintes módulos da PeNSE 2015: características básicas da população do estudo, hábitos alimentares e características do ambiente escolar ${ }^{7}$.
A variável dependente deste estudo foi a prevalência de baixa adesão à alimentação escolar. Esta foi construída a partir da variável do banco de dados gerada pela questão "Você costuma comer a comida (merenda/almoço) oferecida pela escola? (não considerar lanches/comida comprados na cantina)", cujas respostas poderiam ser: (a) "sim, todos os dias"; (b) "sim, 3 a 4 dias por semana"; (c) "sim, 1 a 2 dias por semana"; (d) "raramente"; e (e) "não". Posteriormente, essas respostas foram categorizadas em: (1) baixa adesão ( $\leq 2$ dias/semana) à alimentação escolar considerando as respostas (c), (d) e (e); e (0) média ou alta adesão ( $\geq 3$ dias/semana) da alimentação escolar, considerando as respostas (a) e (b).

As variáveis independentes se referiram ao nível individual e ao escolar. Para o nível individual, foram consideradas variáveis: sociodemográficas; de estado nutricional; de consumo alimentar; de rotina alimentar; de comportamentos relacionados à saúde; de características da família. Discute-se que as questões alimentares e nutricionais têm escopo complexo, e por isso, foram utilizadas variáveis que caracterizam o indivíduo quanto seus aspectos físicos, biológicos e comportamentais. Apesar de variáveis como a insatisfação corporal não serem comumente exploradas em estudos de adesão à alimentação escolar, a destacada prevalência dessa questão na população de adolescentes pode representar algum efeito o qual poderá ser avaliado nesse estudo exploratório, como visto para outras práticas alimentares ${ }^{16}$. As variáveis do nível escolar foram dos tipos: características da escola, espaço geográfico; e ambiente alimentar. Esses fatores relacionados ao ambiente alimentar escolar influenciam de forma direta na adesão à alimentação escolar, uma vez que implica, por exemplo, nas escolhas alimentares, no acesso e no conforto para realizar as refeições (Quadro 1).

\section{Análises estatísticas}

Analisou-se a baixa adesão à alimentação escolar por adolescentes considerando as variáveis independentes dos níveis individual e escolar. Para análise descritiva dos dados, as prevalências da variável dependente foram estimadas considerando cada variável independente, assim como seus respectivos Intervalos de Confiança de 95\% (IC95\%). Além disso, foram verificadas se as diferenças de prevalências de baixa adesão à alimentação escolar encontradas tinham significância estatística $(\mathrm{p}<0,05)$ ao ser aplicado o teste qui-quadrado de Pearson. 
Quadro 1. Descrição das variáveis independentes, segundo suas características e dimensões. PeNSE, 2015.

\begin{tabular}{|c|c|}
\hline $\begin{array}{c}\text { Dimensão/característica/ } \\
\text { variável }\end{array}$ & Descrição \\
\hline \multicolumn{2}{|r|}{ Nível individual } \\
\hline \multicolumn{2}{|l|}{ Sociodemográficas } \\
\hline Sexo & Adolescente do sexo feminino ou masculino. \\
\hline Cor/raça & $\begin{array}{l}\text { Adolescente que se auto declarou de cor/raça branca ou não branca (preta, } \\
\text { amarela, parda ou indígena). }\end{array}$ \\
\hline Faixa etária & Adolescente de 10-14 anos ou de 15-19 anos de idade na data da pesquisa. \\
\hline Trabalha & Adolescente com algum trabalho, emprego ou negócio na data da pesquisa. \\
\hline \multicolumn{2}{|l|}{ Familiar } \\
\hline Escolaridade da mãe & $\begin{array}{l}\text { Nível de ensino (grau) da mãe do adolescente no momento da pesquisa: sem } \\
\text { instrução, alfabetizada, ensino fundamental, ensino médio, ensino superior, } \\
\text { estudante não sabe informar. }\end{array}$ \\
\hline $\begin{array}{l}\text { Número de moradores no } \\
\text { domicílio }\end{array}$ & $\begin{array}{l}\text { Adolescente morando em domicílios com cinco ou mais pessoas ou com até } \\
\text { quatro pessoas. }\end{array}$ \\
\hline $\begin{array}{l}\text { Vivência da fome no } \\
\text { domicílio }\end{array}$ & $\begin{array}{l}\text { Adolescente relatou algum episódio fome por falta de comida em casa nos últimos } \\
30 \text { dias. }\end{array}$ \\
\hline \multicolumn{2}{|l|}{ Estado nutricional } \\
\hline Excesso de peso & Adolescente classificados com sobrepeso e obesidade (IMC/I $\geq$ Escore- $z+1)^{17}$. \\
\hline \multicolumn{2}{|l|}{ Consumo alimentar } \\
\hline Consumo regular de feijão & Consumo de feijão em cinco ou mais dias da semana. \\
\hline $\begin{array}{l}\text { Consumo regular de } \\
\text { legumes e verduras }\end{array}$ & $\begin{array}{l}\text { Consumo de legume ou verdura em cinco ou mais dias na semana. Exemplos: } \\
\text { alface, abóbora, brócolis, cebola, cenoura, chuchu, couve, espinafre, pepino, } \\
\text { tomate etc. Não incluiu batata e aipim (mandioca/macaxeira). }\end{array}$ \\
\hline Consumo regular de frutas & Consumo de frutas frescas ou salada de frutas em cinco ou mais dias na semana. \\
\hline $\begin{array}{l}\text { Consumo regular de } \\
\text { salgados fritos }\end{array}$ & $\begin{array}{l}\text { Consumo de salgados fritos em cinco ou mais dias na semana. Exemplo: batata } \\
\text { frita (sem contar a batata de pacote) ou salgados fritos como coxinha de galinha, } \\
\text { quibe frito, pastel frito, acarajé etc. }\end{array}$ \\
\hline $\begin{array}{l}\text { Consumo regular de } \\
\text { guloseimas }\end{array}$ & $\begin{array}{l}\text { Consumo de guloseimas (doces, balas, chocolates, chicletes, bombons ou } \\
\text { pirulitos) em cinco ou mais dias na semana. }\end{array}$ \\
\hline $\begin{array}{l}\text { Consumo regular de } \\
\text { refrigerantes }\end{array}$ & Consumo de refrigerantes em cinco ou mais dias na semana. \\
\hline $\begin{array}{l}\text { Consumo regular } \\
\text { de alimentos } \\
\text { ultraprocessados salgados }\end{array}$ & $\begin{array}{l}\text { Consumo de alimentos industrializados/ultraprocessados salgados (hambúrguer, } \\
\text { presunto, mortadela, salame, linguiça, salsicha, macarrão instantâneo, salgadinho } \\
\text { de pacote, biscoitos salgados) em cinco ou mais dias na semana. }\end{array}$ \\
\hline \multicolumn{2}{|l|}{ Rotina alimentar } \\
\hline $\begin{array}{l}\text { Consumo regular de café } \\
\text { da manhã }\end{array}$ & Consumo de café da manhã em cinco ou mais dias na semana. \\
\hline $\begin{array}{l}\text { Consumo regular de } \\
\text { almoço ou jantar com } \\
\text { responsáveis }\end{array}$ & $\begin{array}{l}\text { Consumo de almoço ou jantar com mãe, pai ou responsável em cinco ou mais } \\
\text { dias na semana. }\end{array}$ \\
\hline $\begin{array}{l}\text { Come enquanto assiste à } \\
\text { televisão ou estuda }\end{array}$ & $\begin{array}{l}\text { Consumo de alimentos enquanto assisti à TV ou estuda em cinco ou mais dias na } \\
\text { semana. }\end{array}$ \\
\hline $\begin{array}{l}\text { Comeu em fast food na } \\
\text { última semana }\end{array}$ & $\begin{array}{l}\text { Consumo de alimentos em restaurantes fast food (lanchonetes, barracas de } \\
\text { cachorro-quente, pizzaria etc) em cinco ou mais dias na semana. }\end{array}$ \\
\hline \multicolumn{2}{|c|}{ Comportamentos relacionados à saúde } \\
\hline Satisfação corporal & $\begin{array}{l}\text { Satisfação do adolescente com seu próprio corpo na data da pesquisa: satisfeito/ } \\
\text { muito satisfeito, indiferente, insatisfeito/muito insatisfeito }\end{array}$ \\
\hline Insuficientemente ativo & $\begin{array}{l}\text { Adolescente que não praticou atividade física ou a praticou por menos de } 300 \\
\text { minutos por semana. }\end{array}$ \\
\hline
\end{tabular}


Quadro 1. Descrição das variáveis independentes, segundo suas características e dimensões. PeNSE, 2015.

\begin{tabular}{|l|l|}
\hline $\begin{array}{l}\text { Dimensão/característica/ } \\
\text { variável }\end{array}$ & \multicolumn{1}{c|}{ Nível escolar } \\
\hline \multicolumn{2}{|c|}{ Característica da escola } \\
\hline Situação da escola & Escola que o adolescente estudava estava localizada em área urbana ou rural. \\
\hline Porte da escola & $\begin{array}{l}\text { Porte da escola adolescente estudava com base no número de alunos matriculados: } \\
\text { até 500, de 500 a 1000, e mais de 1000. }\end{array}$ \\
\hline Macrorregião & $\begin{array}{l}\text { Macrorregião geográfica brasileira onde estava localizada a escola que o } \\
\text { adolescente estudava na data da pesquisa: Norte, Nordeste, Sudeste, Sul, Centro- } \\
\text { Oeste. }\end{array}$ \\
\hline Ambiente Alimentar & Presença de cozinha na escola que o adolescente estudava. \\
\hline Presença de cozinha & Presença de refeitório na escola que o adolescente estudava. \\
\hline Presença de refeitório & Presença de cantina na escola que o adolescente estudava. \\
\hline Presença de cantina & $\begin{array}{l}\text { Presença de ponto alternativo de venda de alimentos na escola que o adolescente } \\
\text { estudava. }\end{array}$ \\
\hline $\begin{array}{l}\text { Presença de ponto } \\
\text { alternativo de venda }\end{array}$ & Presença de horta na escola que o adolescente estudava. \\
\hline Presença de horta &
\end{tabular}

Técnicas de regressão multinível de Poisson foram empregadas para verificar o efeito das variáveis dos níveis individual e escolar na baixa adesão à alimentação escolar por adolescentes (variável dependente). Inicialmente, foi estimado um modelo de interceptos aleatórios (modelo nulo) para avaliar a possibilidade de realização da análise multinível. Essa técnica teve como objetivo verificar se a variável dependente apresentava uma distribuição diferente considerando o nível escolar. Após verificar a viabilidade dessa análise, regressões multiníveis de Poisson bivariadas estimaram as Razões de Prevalência (RP) brutas e seus respectivos IC $95 \%$. Foram consideradas as melhores situações como categorias de referência de cada uma das variáveis independentes do nível individual e do nível escolar. Essas foram incluídas uma a uma no modelo nulo inicial. Tal etapa foi realizada para observar se havia associação significativa entre cada variável independente e a dependente considerando o efeito do contexto escolar.

Posteriormente, as variáveis de nível individual que apresentaram $p<0,20$ nas análises bivariadas foram testadas com relação aos coeficientes e interceptos. Todas eram do tipo coeficientes fixos e interceptos aleatórios e foram adicionadas ao modelo múltiplo, gerando RP ajustadas e seus respectivos IC95\%. Essa etapa gerou o modelo 1 composto por variáveis do nível individual que permaneceram com significância estatística $(\mathrm{p}<0,05)$. Finalmente, foi estimado o modelo 2 , composto por variáveis do nível escolar significativas na análise bivariada, junto a todas as variáveis independentes do modelo 1 , gerando RP ajustadas para o nível individual e o nível escolar e seus respectivos IC95\%. A qualidade dos modelos foi avaliada pela estimativa e observação da modificação da variância e da significância do $L i$ kelihood Ratio test (LRtest). O modelo final (modelo 2) foi alcançado quando as variáveis foram adicionadas e o modelo teve redução de variância e permaneceu significativo $(\mathrm{p}<0,05)$. A estimativa do modelo 2 com a inclusão das variáveis do contexto escolar permitiu verificação do efeito desse contexto sobre a variável dependente, e, também, a identificação dos efeitos mitigadores ou acentuadores do contexto escolar nas RP das variáveis individuais do modelo 1.

Todas as análises foram realizadas no programa Stata versão 13.0 (StataCorp LP, College Station, Estados Unidos) e apenas a análise descritiva considerou o desenho amostral da pesquisa, pois o banco de microdados da PeNSE não disponibiliza os fatores para o nível escolar.

\section{Resultados}

Observou-se que, dos 10.262 alunos analisados, $50,1 \%$ eram do sexo feminino; $66,2 \%$, não brancos (pretos, pardos, indígenas); e 54,3\% tinham 15 anos de idade ou mais. Quanto ao consumo da alimentação escolar, 29,5\% (IC95\%: 28,3- 
$30,7 \%)$ responderam que não consumiam a comida oferecida pela escola; $27,4 \%$ (IC95\%: 26,3$28,5 \%$ ) consumiam raramente; $7,3 \%$ (IC95\%: $6,7-8,1 \%)$ consumiam 1 a 2 vezes por semana; 9,5\% (IC95\%: 8,7-10,2\%) consumiam 3 a 4 vezes por semana; e 26,3\% (IC95\%: 25,2-27,5\%) consumiam todos os dias. A prevalência de baixa adesão à alimentação escolar por adolescentes brasileiros foi, portanto, de 64,2\% (IC95\%: 63,065,4\%).

As variáveis significativas na análise bivariada que foram utilizadas na estimativa dos mo- delos multivariados foram: sexo, idade, excesso de peso, consumo de feijão, consumo de legumes e verduras, consumo de frutas, de salgados fritos, de guloseimas, consumo de refrigerante, consumo de café da manhã, satisfação corporal, escolaridade materna, número de moradores no domicílio, vivência da fome no domicílio (Tabela 1); e situação da escola, porte da escola, presença de cantina, de ponto alternativo de venda de alimentos e de horta (Tabela 2).

Preliminarmente, a variável dependente foi ajustada em um modelo nulo para verificar

Tabela 1. Distribuição e associações bivariadas da baixa adesão à alimentação escolar entre adolescentes brasileiros de escolas públicas e variáveis do nível individual. Pesquisa Nacional de Saúde do Escolar, 2015.

\begin{tabular}{|c|c|c|c|c|c|}
\hline \multirow{2}{*}{ Variáveis } & \multicolumn{5}{|c|}{ Baixa adesão à alimentação escolar } \\
\hline & $\mathbf{n}$ & $\%$ & IC $(95 \%)$ & p-valor & RP (IC 95\%) \\
\hline \multicolumn{6}{|l|}{ Nível individual } \\
\hline \multicolumn{6}{|l|}{ Sociodemográficas } \\
\hline Sexo & & & & 0,001 & \\
\hline Masculino & 5058 & 62,1 & $60,3-63,8$ & & Ref \\
\hline Feminino & 5204 & 66,3 & $64,6-68,0$ & & $1,09(1,04-1,14)^{*}$ \\
\hline Cor/raça & & & & 0,012 & \\
\hline Branca & 3698 & 66,3 & $64,3-68,3$ & & Ref \\
\hline Não branca & 6549 & 63,1 & $61,5-64,6$ & & $0,98(0,93-1,04)$ \\
\hline Idade & & & & $<0,001$ & \\
\hline 10-14 anos & 5704 & 61,3 & $59,6-62,9$ & & Ref \\
\hline 15-19 anos & 4558 & 66,7 & $64,9-68,4$ & & $1,11(1,05-1,18)^{*}$ \\
\hline Trabalha & & & & 0,403 & \\
\hline Não & 8483 & 64,5 & $63,1-65,8$ & & Ref \\
\hline Sim & 1775 & 63,1 & $60,0-66,0$ & & $0,99(0,93-1,06)$ \\
\hline \multicolumn{6}{|l|}{ Estado nutricional } \\
\hline Excesso de peso & & & & 0,022 & \\
\hline Não & 7451 & 63,4 & $61,9-64,8$ & & Ref \\
\hline Sim & 2810 & 66,6 & $64,2-68,9$ & & $1,05(0,99-1,11)^{\star}$ \\
\hline \multicolumn{6}{|l|}{ Consumo alimentar } \\
\hline Consumo regular de feijão & & & & 0,038 & \\
\hline Não & 4163 & 65,8 & $63,9-67,7$ & & Ref \\
\hline Sim & 6064 & 63,1 & $61,5-64,7$ & & $0,96(0,91-1,01)^{*}$ \\
\hline Consumo regular de legumes e verduras & & & & 0,087 & \\
\hline Não & 6238 & 65,4 & $62,5-65,1$ & & Ref \\
\hline Sim & 4017 & 62,2 & $63,6-70,2$ & & $1,06(0,98-1,13)^{\star}$ \\
\hline Consumo regular de frutas & & & & 0,882 & \\
\hline Não & 6756 & 64,2 & $62,6-65,7$ & & Ref \\
\hline Sim & 3492 & 64,4 & $62,2-66,5$ & & $0,96(0,91-1,01)^{*}$ \\
\hline Consumo regular de salgados fritos & & & & 0,087 & \\
\hline Não & 8882 & 63,8 & $62,5-65,1$ & & Ref \\
\hline Sim & 1367 & 67 & $63,6-70,2$ & & $1,06(0,98-1,13)^{*}$ \\
\hline
\end{tabular}


Tabela 1. Distribuição e associações bivariadas da baixa adesão à alimentação escolar entre adolescentes brasileiros de escolas públicas e variáveis do nível individual. Pesquisa Nacional de Saúde do Escolar, 2015.

\begin{tabular}{|c|c|c|c|c|c|}
\hline \multirow{2}{*}{ Variáveis } & \multicolumn{5}{|c|}{ Baixa adesão à alimentação escolar } \\
\hline & n & $\%$ & $\mathrm{IC}(95 \%)$ & p-valor & RP (IC 95\%) \\
\hline Consumo regular de guloseimas & & & & 0,018 & \\
\hline Não & 6399 & 63,0 & $61,4-64,5$ & & Ref \\
\hline Sim & 3848 & 66,1 & $64,1-68,1$ & & $1,05(0,99-1,10)^{*}$ \\
\hline Consumo regular refrigerantes & & & & $<0,001$ & \\
\hline Não & 7547 & 62,2 & $60,9-63,8$ & & Ref \\
\hline Sim & 2701 & 69,2 & $66,9-71,5$ & & $1,09(1,03-1,15)^{*}$ \\
\hline Consumo regular de ultraprocessados salgados & & & & 0,201 & \\
\hline Não & 7276 & 63,7 & $62,2-65,2$ & & Ref \\
\hline Sim & 2963 & 65,5 & $63,2-67,7$ & & $1,03(0,98-1,09)$ \\
\hline \multicolumn{6}{|l|}{ Rotina alimentar } \\
\hline Consumo regular de café da manhã & & & & 0,007 & \\
\hline Sim & 4781 & 62,5 & $60,6-64,3$ & & Ref \\
\hline Não & 5477 & 65,9 & $64,2-67,5$ & & $1,05(0,99-1,10)^{*}$ \\
\hline $\begin{array}{l}\text { Consumo regular de almoço ou jantar com } \\
\text { responsáveis }\end{array}$ & & & & $<0,001$ & \\
\hline Sim & 7565 & 62,2 & $60,7-63,6$ & & Ref \\
\hline Não & 2687 & 69 & $66,7-71,3$ & & $1,11(1,05-1,17)^{*}$ \\
\hline Come enquanto assiste à televisão ou estuda & & & & $0,007 ?$ & \\
\hline Não & 5477 & 65,9 & $64,2-67,5$ & & Ref \\
\hline Sim & 4781 & 62,5 & $60,6-64,3$ & & $0,96(0,91-1,00)^{*}$ \\
\hline Comeu em fast food na última semana & & & & 0,125 & \\
\hline Não & 5756 & 63,4 & $61,7-65,0$ & & Ref \\
\hline Sim & 4491 & 65,3 & $63,4-67,2$ & & $1,02(0,98-1,08)$ \\
\hline \multicolumn{6}{|l|}{ Comportamentos relacionados à saúde } \\
\hline Satisfação corporal & & & & 0,001 & \\
\hline Satisfeito/Muito satisfeito & 7393 & 62,8 & $61,3-64,2$ & & Ref \\
\hline Indiferente & 1030 & 66,9 & $62,9-70,7$ & & $1,07(0,99-1,16)^{*}$ \\
\hline Insatisfeito/Muito insatisfeito & 1726 & 68,7 & $65,8-71,6$ & & $1,10(1,03-1,17)^{*}$ \\
\hline \multicolumn{6}{|l|}{ Medidas gerais de saúde } \\
\hline Insuficientemente ativo & & & & 0,544 & \\
\hline Não & 2123 & 63,4 & $60,6-66,1$ & & Ref \\
\hline Sim & 8073 & 64,3 & $62,9-65,7$ & & $1,03(0,97-1,10)$ \\
\hline \multicolumn{6}{|l|}{ Características familiares } \\
\hline Escolaridade da mãe & & & & $<0,001$ & \\
\hline Sem instrução & 538 & 55,9 & $50,0-61,7$ & & Ref \\
\hline Alfabetizada & 2163 & 61,6 & $58,8-64,2$ & & $1,11(0,98-1,26)^{*}$ \\
\hline Ensino fundamental & 1442 & 62,4 & $59,0-65,7$ & & $1,16(1,02-1,33)^{*}$ \\
\hline Ensino médio & 2303 & 67,5 & $65,0-69,9$ & & $1,21(1,06-1,37)^{\star}$ \\
\hline Ensino Superior & 1005 & 64,6 & $60,5-68,4$ & & $1,17(1,02-1,35)^{*}$ \\
\hline Não sabe & 2797 & 66,4 & $64,0-68,7$ & & $1,21(1,07-1,37)^{*}$ \\
\hline Número de moradores no domicílio & & & & $<0,001$ & \\
\hline 5 ou mais & 4325 & 61,2 & $59,2-63,1$ & & Ref \\
\hline até 4 & 5933 & 66,3 & $64,7-67,9$ & & $1,08(1,03-1,14)^{*}$ \\
\hline Vivência da fome no domicílio & & & & 0,046 & \\
\hline Sim & 7979 & 64,9 & $63,5-66,3$ & & Ref \\
\hline Não & 2272 & 61,8 & $59,1-64,5$ & & $0,95(0,90-1,01)^{*}$ \\
\hline
\end{tabular}

${ }^{\star}$ Associações bivariadas com $\mathrm{p}<0,20$. 
o efeito do contexto escolar, a partir do qual o teste da razão de probabilidade (LRtest $=58,74$ ) identificou efeito significativo $(\mathrm{p}<0,001)$ (Tabela 3). Quando foi estimado o modelo multinível incluindo somente variáveis do nível individual (modelo 1), que foram significativas na análise bivariada, perderam significância: o excesso de peso, o consumo de feijão, legumes e verduras, frutas, salgados fritos, guloseimas, o consumo de café da manhã, a satisfação corporal e a vivência da fome no domicílio (Tabela 3).

Nesse modelo 1, o qual considerou apenas variáveis do contexto individual, foi identificada maior prevalência de baixa adesão à alimentação escolar entre adolescentes do sexo feminino (RP: 1,09; IC95\%: 1,04-1,15), de 15 anos ou mais de idade (RP: 1,11; IC95\%: 1,05-1,18), com consumo regular de refrigerantes (RP: 1,08; IC95\%: 1,03-1,15), que não realizavam regularmente o almoço ou jantar com responsáveis (RP: 1,08; IC95\%: 1,02-1,14), com mães mais escolarizadas - Ensino Fundamental (RP: 1,15; IC95\%: 1,01-1,31), Ensino Médio (RP: 1,18; IC95\%: 1,04-1,34), Ensino Superior (RP: 1,16; IC95\%: 1,01-1,34) - ou que o adolescente não sabia relatar a escolaridade da mãe (RP: 1,21; IC95\%: 1,07$1,37)$; e aqueles vivendo em casas com até 4 moradores (RP: 1,07; IC95\%: 1,02-1,13). O modelo

Tabela 2. Distribuição e associações bivariadas da baixa adesão à alimentação escolar entre adolescentes brasileiros de escolas públicas e variáveis do nível escolar. Pesquisa Nacional de Saúde do Escolar, 2015.

\begin{tabular}{|c|c|c|c|c|c|}
\hline Variáveis & $\mathbf{n}$ & $\%$ & IC $(95 \%)$ & p-valor & RP (IC95\%) \\
\hline \multicolumn{6}{|l|}{ Nível escolar } \\
\hline \multicolumn{6}{|l|}{ Características da escola } \\
\hline Situação da escola & & & & $<0,001$ & \\
\hline Rural & 616 & 51,9 & $46,5-57,3$ & & Ref \\
\hline Urbana & 9646 & 65,0 & $63,7-66,3$ & & $1,30(1,14-1,49)^{*}$ \\
\hline Porte da escola (número de alunos) & & & & $<0,001$ & \\
\hline até 500 & 1964 & 55,5 & $52,7-58,2$ & & Ref \\
\hline de 501 a 1000 & 3743 & 62,0 & $59,8-64,1$ & & $1,16(1,06-1,27)^{*}$ \\
\hline mais de 1000 & 4555 & 70,1 & $68,3-71,9$ & & $1,30(1,19-1,42)^{*}$ \\
\hline Macrorregião & & & & $<0,001$ & \\
\hline Norte & 1804 & 69,2 & $66,6-71,7$ & & Ref \\
\hline Nordeste & 1929 & 60,0 & $57,4-62,4$ & & $0,92(0,82-1,02)$ \\
\hline Sudeste & 2028 & 65,8 & $63,5-68,1$ & & $1,01(0,90-1,12)$ \\
\hline Sul & 2253 & 64,1 & $61,9-66,2$ & & $0,98(0,89-1,09)$ \\
\hline Centro-Oeste & 2248 & 64,9 & $62,6-67,2$ & & $0,97(0,87-1,08)$ \\
\hline Brasil & & 64,2 & $63,0-65,4$ & & \\
\hline \multicolumn{6}{|l|}{ Ambiente Alimentar } \\
\hline Presença de cozinha & & & & 0,124 & \\
\hline Sim & 9962 & 64,3 & $63,1-65,6$ & & Ref \\
\hline Não & 300 & 59,3 & $52,7-65,5$ & & $0,93(0,77-1,12)$ \\
\hline Presença de Refeitório & & & & 0,524 & \\
\hline Sim & 6053 & 63,9 & $62,3-65,5$ & & Ref \\
\hline Não & 4209 & 64,7 & $62,7-66,7$ & & $0,94(0,88-1,01)$ \\
\hline Presença de cantina & & & & $<0,001$ & \\
\hline Não & 5875 & 60,5 & $58,9-62,1$ & & Ref \\
\hline $\operatorname{Sim}$ & 4387 & 69,6 & $67,7-71,4$ & & $1,19(1,11-1,27)^{\star}$ \\
\hline Presença de ponto alternativo de venda & & & & 0,003 & \\
\hline Não & 7115 & 63,0 & $61,4-64,5$ & & Ref \\
\hline Sim & 3147 & 66,9 & $64,7-69,0$ & & $1,06(0,99-1,14)^{*}$ \\
\hline Presença de horta & & & & 0,031 & \\
\hline Sim & 2857 & 61,9 & $59,4-64,3$ & & Ref \\
\hline Não & 7405 & 65,0 & $63,6-66,4$ & & $1,07(0,99-1,15)^{\star}$ \\
\hline
\end{tabular}

*Associações bivariadas com $\mathrm{p}<0,20$. 
1 apresentou efeito (LRtest $=43,62)$ significativo $(<0,001)$ e ajuste da variância $(-17,6 \%)$ em relação ao modelo nulo (Tabela 3 ).

O modelo 2 foi estimado a partir das variáveis individuais do modelo 1 e da inclusão das variáveis de nível escolar com efeito significativo na análise bivariada. Das variáveis que haviam atendido a essa condição, a situação da escola, a presença de ponto alternativo de venda de alimentos e de cantina perderam significância no modelo multinível final. Permaneceram como variáveis do nível escolar no modelo 2: o porte da escola (número de alunos) - de 501 a 1000 (RP:
1,12; IC95\%: 1,03-1,22), mais de 1000 (RP: 1,22; IC95\%: 1,12-1,33); e a presença de cantina - sim (RP: 1,15; IC95\%: 1,08-1,22) (Tabela 3).

Além disso, a escolaridade da mãe, a qual apareceu no modelo 1, perdeu significância estatística (efeito mitigador) quando incluída na modelagem junto às variáveis do nível escolar. Não foram verificados outros efeitos mitigadores ou acentuadores nas variáveis do nível individual após a inclusão das variáveis do contexto escolar. Finalmente, o modelo 2 apresentou efeito (LRtest=27,91) significativo $(\mathrm{p}<0,001)$ e maior ajuste da variância $(-38,2 \%)$ em relação ao modelo nulo e o modelo

Tabela 3. Modelos gerados pela análise multinível de regressão de Poisson com efeitos mistos e seus parâmetros de efeitos fixos e randômicos da baixa adesão à alimentação escolar entre adolescentes brasileiros de escolas públicas. Pesquisa Nacional de Saúde do Escolar, 2015.

\begin{tabular}{|c|c|c|c|c|c|}
\hline \multirow{3}{*}{ Variáveis } & \multicolumn{5}{|c|}{ Modelos } \\
\hline & \multirow{2}{*}{ Modelo Nulo } & \multicolumn{2}{|c|}{ Modelo 1} & \multicolumn{2}{|c|}{ Modelo 2} \\
\hline & & RP (IC 95\%) & p-valor & RP (IC 95\%) & p-valor \\
\hline \multicolumn{6}{|l|}{ Nível escolar } \\
\hline \multicolumn{6}{|c|}{ Porte da escola (número de alunos) } \\
\hline de 501 a 1000 & & & & $1,12(1,03-1,22)$ & 0,010 \\
\hline mais de 1000 & & & & $1,22(1,12-1,33)$ & $<0,0001$ \\
\hline \multicolumn{6}{|l|}{ Presença de cantina } \\
\hline $\operatorname{Sim}$ & & & & $1,15(1,08-1,22)$ & $<0,0001$ \\
\hline \multicolumn{6}{|l|}{ Nível individual } \\
\hline \multicolumn{6}{|l|}{ Sexo } \\
\hline Feminino & & $1,09(1,04-1,15)$ & $<0,001$ & $1,09(1,03-1,14)$ & 0,001 \\
\hline \multicolumn{6}{|l|}{ Idade } \\
\hline 15 anos ou mais & & $1,11(1,05-1,18)$ & $<0,001$ & $1,08(1,02-1,14)$ & 0,009 \\
\hline \multicolumn{6}{|c|}{ Consumo regular de refrigerantes } \\
\hline Sim & & $1,08(1,03-1,15)$ & 0,003 & $1,09(1,03-1,15)$ & 0,003 \\
\hline \multicolumn{6}{|c|}{$\begin{array}{l}\text { Consumo alimentar de almoço ou } \\
\text { jantar com responsáveis }\end{array}$} \\
\hline Não & & $1,08(1,02-1,14)$ & 0,005 & $1,08(1,02-1,14)$ & 0,006 \\
\hline \multicolumn{6}{|l|}{ Escolaridade da mãe } \\
\hline Alfabetizada & & $1,09(0,96-1,24)$ & 0,198 & - & - \\
\hline Ensino fundamental & & $1,15(1,01-1,31)$ & 0,042 & - & - \\
\hline Ensino médio & & $1,18(1,04-1,34)$ & 0,012 & - & - \\
\hline Ensino Superior & & $1,16(1,01-1,34)$ & 0,040 & - & - \\
\hline Não sabe & & $1,21(1,07-1,37)$ & 0,003 & - & - \\
\hline \multicolumn{6}{|l|}{ Número de moradores } \\
\hline até 4 & & $1,07(1,02-1,13)$ & 0,006 & $1,07(1,02-1,13)$ & 0,006 \\
\hline \multicolumn{6}{|l|}{ Efeitos fixos } \\
\hline Intercepto (IC95\%) & $-0,475(-0,509-(-0,440))$ & $0,454(0,39$ & $9-0,515)$ & $0,442(0,4$ & $5-0,481)$ \\
\hline Efeitos Randômicos & Variância (SE) & Variât & cia (SE) & Vari & ncia (SE) \\
\hline Nível escolar & $0,034(0,023-0,051)$ & $0,028(0,01$ & $8-0,044)$ & $0,021(0,0$ & $3-0,036)$ \\
\hline Variação & & & $-17,6$ & & $-38,2$ \\
\hline LR Test (Chi2; p-valor) & $58,74(<0,001)$ & 43,62 & $<0,001)$ & 27,91 & $\mathrm{p}<0,001)$ \\
\hline
\end{tabular}

IC = Intervalo de confiança; LR = Likelihood Ratio. 
1. Isso sugere que essa modelagem, considerando o contexto individual e escolar, é mais apropriada para compreensão da determinação da variável dependente. Tal resultado destaca, portanto, que os principais fatores implicados na baixa adesão à alimentação escolar entre adolescentes brasileiros são os de contexto escolar: porte da escola e presença de cantina (Tabela 3 ).

\section{Discussão}

No presente estudo, foi identificada elevada prevalência de baixa adesão à alimentação escolar entre adolescentes brasileiros. Esse problema se associou expressivamente aos determinantes do contexto escolar: porte da escola e presença de cantina; e aos determinantes individuais: sexo feminino, idade superior a 15 anos, consumo regular de refrigerantes, prática irregular de almoçar ou jantar com os responsáveis e o menor número de moradores no domicílio do adolescente.

Os determinantes individuais da adesão à alimentação escolar como fatores sociodemográficos, de consumo e de rotina alimentar foram identificados em outros estudos. Tais resultados corroboram os efeitos do estudo, que avaliou a regularidade do consumo de alimentação escolar a partir dos dados da PeNSE 2012. Neste, foi estimado que apenas $22 \%$ dos adolescentes consumiam regularmente as refeições ofertadas pelas escolas, sendo essa prática associada aos alunos do sexo masculino, pardos, que não moravam em capitais, com mães com baixa escolaridade, e que tomavam café da manhã ou almoçavam com os pais ${ }^{9}$.

Observa-se, dessa forma, apenas algumas diferenças entre as variáveis individuais que compuseram o modelo final do presente estudo e do estudo com dados da PeNSE 2012. Neste estudo, no qual foram utilizados os dados da PeNSE 2015, a inclusão das variáveis do contexto escolar em modelagem multinível mitigou as variáveis da cor/raça, escolaridade materna, da prática de tomar café da manhã e da localização geográfica. Provavelmente, pela homogeneidade da posição socioeconômica ocupada pelos adolescentes de escolas públicas, a qual interfere sobre as rotinas alimentares familiares, como o caso da irregularidade nas refeições em família, além do importante efeito das variáveis de contexto nesse comportamento alimentar de baixa adesão à alimentação escolar.

No ambiente escolar, sabe-se que a opção por alimentos comercializados nas cantinas em detrimento dos ofertados pela escola se associa ao maior consumo de alimentos processados e ultraprocessados ${ }^{12,13}$. Esse grupo de alimentos marcadores de dietas não saudáveis (guloseimas, refrigerantes e biscoitos doces) é um componente relevante no consumo alimentar de adolescentes brasileiros de escolas públicas e privadas ${ }^{4}$.

A presença de cantina na escola se constituiu como um determinante no modelo de baixa adesão à alimentação escolar. Esses espaços que comercializam alimentos competitivos nesse ambiente se somam aos fatores socioeconômicos e às preferências alimentares dos alunos e acarretam o consumo irregular das refeições do PNAE ${ }^{11}$. Além disso, comer na cantina da escola parece unir as preferências alimentares dos adolescentes ao melhor status social. Nesse sentido, um estudo qualitativo apontou que o medo de adquirir uma "identidade de pobreza e inferioridade" pode ser uma das explicações para o consumo irregular, por parte dos adolescentes, da alimentação servida em escolas públicas ${ }^{18}$.

A persistência desse tipo de comércio nas escolas públicas brasileiras pode ser explicada pelo número limitado de iniciativas municipais e estaduais que regulamentam a comercialização de alimentos ultraprocessados nesses espaços, acrescido à deficiência na fiscalização da execução dessas normativas, quando existentes ${ }^{19,20}$. Provavelmente, a maior adesão à alimentação escolar por parte dos adolescentes depende de um conjunto de ações, as quais devem ser pautadas na educação alimentar e nutricional como processo permanente de ensino e aprendizagem e outras estratégias para promoção da alimentação adequada e saudável nas escolas ${ }^{21,22}$.

Outro estudo qualitativo, que analisou a percepção de estudantes adolescentes baianos sobre a alimentação escolar, identificou que algumas preparações, que eram parte da cultura alimentar local e faziam parte do conjunto de refeições servidas em casa, não eram bem aceitas no espaço escolar, ambiente de comida leve e com característica de "lanche". Isso destacou que "a ênfase colocada na percepção dos escolares sobre o comer na escola confirma que estes possuem uma cultura alimentar e são também influenciados por novidades do mercado consumidor e pela propaganda" 23 .

Uma investigação com estudantes do interior da Bahia verificou que a quebra de estigma com relação à alimentação escolar, a qual acarretava a melhor adesão, dava-se pelo desenvolvimento de uma identidade de compartilhamento da comida. Para isso, a participação de professores e 
gestores das refeições no ambiente comum aos alunos gerava maior confiança desses em relação à qualidade e à importância dos alimentos servidos $^{24}$.

Deve-se considerar que essas práticas alimentares dos adolescentes, sejam na dimensão do consumo ou da rotina alimentar, são muito influenciadas pela expansão da indústria de alimentos $^{25}$. Os espaços de comercialização de alimentos ultraprocessados estão presentes nas escolas e em suas proximidades, constituindo-se como um risco para a formação de padrões alimentares inadequados às recomendações de promoção da saúde ${ }^{12,14}$.

Tudo isso mostra a necessidade de intensificar as investigações sobre o papel do ambiente alimentar da escola na determinação das práticas alimentares dos adolescentes. Passo fundamental ao desenvolvimento de estratégias que reduzam riscos à saúde desse grupo etário, principalmente, baseadas em ações efetivas para melhoria da qualidade das refeições servidas, dos espaços para consumo e dos alimentos comercializados na escola $^{18,26,27}$.

Destaca-se, com isso, a atual discussão sobre ambientes obesogênicos e a necessidade, por exemplo, de regulação do comércio de alimentos nas escolas, defendida no Plano de Ação para a Prevenção da obesidade em crianças e adolescentes OPAS/OMS ${ }^{28}$. A comercialização de alimentos influencia o aumento da prevalência de consumo de ultraprocessados, dos quais se destacam os refrigerantes, identificados como um dos determinantes da baixa adesão à alimentação escolar no presente estudo. Esse é um exemplo de produto bastante consumido por adolescentes, seja no lanche ou em refeições principais ${ }^{29,30}$, e seu alto consumo e preferência é influenciado, também, pela regulação pouco eficiente das indústrias alimentícias e de suas propagandas no Brasil ${ }^{31}$.

Deve-se discutir que esses ambientes que geram práticas e comportamentos alimentares não são apenas obesogênicos, mas também ambientes de risco para alimentação e saúde. As recomendações para transformação dos ambientes obesogênicos são estratégias que não estão restritas apenas ao controle de um agravo (obesidade), e sim para promoção da saúde de forma ampliada ${ }^{32}$. Os resultados do presente estudo contribuem com a expansão da discussão sobre os ambientes alimentares ao identificar a presença de cantinas como um determinante da baixa adesão a um Programa que tem como diretrizes a cobertura universal dos estudantes para promoção de hábitos alimentares saudáveis. Essa ação é, por isso, de garantia da segurança alimentar e nutricional, e não apenas de combate a um desvio nutricional ${ }^{3}$.

Os desafios de efetivação do PNAE se relacionam a preferências alimentares dos estudantes, mas também às possíveis dificuldades de gestão do Programa na escola. A baixa adesão à alimentação escolar é influenciada, também, pela oferta de cardápios pouco variados devido às dificuldades de gestão frente ao pouco recurso financeiro, mão de obra insuficiente, cardápios ${ }^{13}$.

O porte da escola, neste estudo, é avaliado pelo número de alunos, o qual foi relevante no modelo final pode representar um proxy da execução do PNAE. Sabe-se, na prática, que um maior número de alunos dificulta a execução do cardápio devido à baixa disponibilidade de recursos financeiros. Além disso, a deficiência na quantidade de recursos humanos (nutricionistas e cozinheiras) para o planejamento e execução de um cardápio de qualidade, e até mesmo a logística no momento da distribuição das preparações aos estudantes pode gerar mais tempo, proporcionando filas que, desse modo, desmotivam os adolescentes a consumirem as refeições servidas.

Outras hipóteses podem ser levantadas, como as destacadas nos resultados de um estudo com adolescentes de escolas públicas da Bahia, no qual os estudantes entendiam como incompatíveis com o momento do "recreio" os tipos de cardápios servidos (sopas e mingaus). Os resultados da análise qualitativa deste estudo destacaram que os alunos descreviam o momento do "recreio" como espaço das brincadeiras e socialização e, por isso, seria um momento de comer "besteira", fazendo referência a alimentos ultraprocessados, como salgados de pacotes, biscoitos, balas e doces ${ }^{23}$.

Nessa perspectiva, o incentivo à adesão da alimentação escolar pelos adolescentes precisa ser trabalhado. Sabe-se que é durante a adolescência que o indivíduo começa a estabelecer suas identidades, experimentando formas de posicionar-se diante do mundo. As maneiras de comer e o tipo de comida também são um marcador de pertencimento identitário, muito influenciado por modos de viver a vida pautada na transgressão a normas sociais ${ }^{33}$, não sendo diferente com a alimentação. Sendo assim, o PNAE precisa ser executado junto a escolas que atendem adolescentes, considerando, mais ainda, suas dimensões da oferta de refeições adequadas, além da educação alimentar e nutricional ${ }^{3}$. Esta deve ser trabalhada buscando o desenvolvimento crítico e reflexivo dos adolescentes para realização de escolhas alimentares mais saudáveis de forma autônoma ${ }^{34,35}$. 
Os resultados apontam, também, para relação da baixa adesão à alimentação escolar com a rotina alimentar dos adolescentes, e associações semelhantes foram identificadas a partir de dados da PeNSE 2012, na qual o consumo irregular de alimentação escolar foi menor entre os adolescentes que tomavam café da manhã e almoçavam com os pais regularmente ${ }^{9}$. Apesar do presente estudo só ter encontrado associação da baixa adesão com o consumo irregular de almoço/jantar, essa baixa frequência de realização de refeições familiares em família reforça a importância dessa rotina para nutrição e condições de saúde dos indivíduos ${ }^{36}$. Provavelmente, esse aspecto explica a baixa adesão ao PNAE entre adolescentes que não fazem refeições regularmente com seus responsáveis, por não serem orientados da importância da refeição servida na escola e de uma alimentação saudável. As refeições familiares se constituem como prática promotora do estreitamento de vínculos familiares e de educação alimentar e nutricional ${ }^{34,37,38}$.

Ainda no contexto familiar do adolescente, os resultados, evidenciando associações entre o menor número de moradores no domicílio e a irregularidade do consumo da alimentação escolar, demonstram relações entre a maior quantidade de pessoas da família e as piores situações de alimentação, nutrição e saúde. Um inquérito que investigou insegurança alimentar, por exemplo, estimou maiores prevalências de insegurança alimentar moderada e grave entre adolescentes que residiam com mais de 4 indivíduos ${ }^{39}$. Logo, pode-se considerar o número de moradores de uma residência como um proxy da condição financeira do Brasil, no qual adolescentes que vivem em residências com menos moradores, geralmente, apresentam melhores condições de vida do que aqueles que dividem o espaço com mais pessoas. Esse fato também pode estar relacionado ao fato de adolescentes com melhores condições financeiras optarem por comprar a alimentação que irão consumir na escola.
A inexistência de dados sobre características relacionadas à qualidade nutricional das refeições servidas nas escolas, os quais não foram coletados nos estudos da PeNSE, consiste em uma limitação deste estudo. Principalmente, porque não se pode investigar relações entre a qualidade das refeições servidas e a baixa adesão à alimentação escolar. Resultados de um estudo com crianças de São Paulo ressaltaram a importância de compreender a aceitação das refeições no contexto da adesão à alimentação escolar. Seus achados demonstram que a inserção de preparações adequadas à cultura e aos hábitos alimentares dos estudantes no cardápio podem aumentar a aceitabilidade dos alimentos ofertados ${ }^{40}$. Apesar dessa limitação da PeNSE, o estudo possui grande validade interna e externa por ser representativo de toda população de adolescentes de escolas públicas do Brasil. Outra potencialidade desta investigação centra-se na identificação de determinantes individuais e da escola associados à baixa adesão à alimentação escolar com o emprego de técnica adequada de análise para esses níveis.

Desse modo, a baixa adesão à alimentação escolar caracteriza-se como marcador de risco para alimentação saudável e nutrição adequada entre adolescentes de escolas públicas brasileiras. A elevada prevalência dessa baixa adesão às refeições do PNAE identificada entre esse grupo etário parece ser determinada por dimensões da gestão da escola, do ambiente alimentar escolar, do ambiente familiar, da rotina alimentar, do consumo alimentar e do comportamento individual do adolescente. Esses resultados devem ser aprofundados em outros estudos, pois são importantes para reprogramação da execução desse importante programa de segurança alimentar e nutricional e das ações de promoção da alimentação saudável em escolas públicas do Brasil que atendem esse público. 


\section{Colaboradores}

D Vale e AG Roncalli contribuíram para o desenho, análise e interpretação de dados; redação do artigo ou revisão crítica relevante ao conteúdo intelectual; aprovação final da versão a ser publicada. CO Lyra, TT Santos e CVS Souza contribuíram para a análise e interpretação de dados; Revisão crítica relevante para conteúdo intelectual; aprovação final da versão a ser publicada.

\section{Referências}

1. Brasil. Decreto $\mathrm{n}^{\circ} 7.272$, de 25 de agosto de 2010 . Regulamenta a Lei $\mathrm{n}^{\circ} 11.346$, de 15 de setembro de 2006, que cria o Sistema Nacional de Segurança Alimentar e Nutricional - SISAN com vistas a assegurar o direito humano à alimentação adequada, institui a Política Nacional de Segurança Alimentar e Nutricional - PNSAN, estabelece os parâmetros para a elaboração do Plano Nacional de Segurança Alimentar e Nutricional, e dá outras providências. Diário Oficial da União 2010; 26 ago.

2. Brasil. Ministério da Saúde (MS). Política Nacional de Alimentação e Nutrição. Brasília: MS; 2012.

3. Brasil. Lei no 11.947, de 16 de junho de 2009. Dispõe sobre o atendimento da alimentação escolar e do Programa Dinheiro Direto na Escola aos alunos da educação básica; altera as Leis no 10.880 , de 9 de junho de 2004, no 11.273, de 6 de fevereiro de 2006, n 11.507, de 20 de julho de 2007; revoga dispositivos da Medida Provisória no 2.178-36, de 24 de agosto de 2001, e a Lei no 8.913 , de 12 de julho de 1994; e dá outras providências. Diário Oficial da União 2009; 17 jun.

4. Azeredo C, Resende L, Canella D, Claro RM, Castro I, Luiz O, Levy RB. Dietary intake of Brazilian adolescentes. Public Health Nutr 2015; 18(7):1215-1224.

5. Tavares LF, Castro IRR, Levy RB, Cardoso LO, Claro RM. Padrões alimentares de adolescentes brasileiros: resultados da Pesquisa Nacional de Saúde do Escolar (PeNSE). Cad Saude Publica 2014; 30(12):2679-2690.

6. Instituto Brasileiro de Geografia e Estatística (IBGE). Coordenação de Indicadores Sociais. Pesquisa nacional de saúde do escolar: 2012. Rio de Janeiro: IBGE; 2013.

7. Instituto Brasileiro de Geografia e Estatística (IBGE). Coordenação de Indicadores Sociais. Pesquisa nacional de saúde do escolar: 2015. Rio de Janeiro: IBGE; 2016.

8. Brasil. Ministério da Educação (MS). Scarparo ALS, Bratkowski GR, organizadoras. Manual para aplicação dos testes de aceitabilidade no Programa Nacional de Alimentação Escolar (PNAE). 2a ed. Brasília: MS; 2017.

9. Locatelli NT, Canella DS, Bandoni DH. Fatores associados ao consumo da alimentação escolar por adolescentes no Brasil: resultados da PeNSE 2012. Cad Saude Publica 2017; 33(4):e00183615.

10. Locatelli NT, Canella DS, Bandoni DH. Positive influence of school meals on food consumption in Brazil. Nutrition 2018; 53:140-144.

11. Cesar JT, Valentim EA, Almeida CCB, Schieferdecker MEM, Schmidt ST. Alimentação Escolar no Brasil e Estados Unidos: uma revisão integrativa. Cien Saude Colet 2018; 23(3):991-1007.

12. Azeredo CM, Rezende LFM, Canella DS, Claro RF, Peres MFT, Luiz OC, França-Junior I, Kinra S, Hawkesworth S, Levy RB. Food environments in schools and in the immediate vicinity are associated with unhealthy food consumption among Brazilian adolescents. Preven Med 2016; 88:73-79.

13. Tiborski CJ, Augusto TC, Maria OM, Teresinha SS. Adesão à alimentação escolar e fatores associados em adolescentes de escolas públicas na região Sul do Brasil. Cien Saude Colet 2020; 25(3):977-988.

14. Carmo AS, Assis MM, Cunha CF, Oliveira TRPR, Mendes LL. The food environment of Brazilian public and private schools. Cad Saúde Pública 2018; 34(12):e00014918. 
15. Brasil. Ministério da Saúde (MS). Conselho Nacional de Saúde (CNS). Resolução no 510, de 7 de abril de 2016. Diário Oficial da União 2016; 24 mar.

16. Carvalho GX, Nunes APN, Moraes CL, Veiga GV. Insatisfação com a imagem corporal e fatores associados em adolescentes. Cien Saude Colet 2020; 25(7):27692782.

17. World Health Organization (WHO). WHO child growth standards: Length/height-for-age, weight-forage, weight-for-length, weight-for-height and body mass index-for-age. Methods and development. Geneva: WHO; 2006.

18. Silva EO, Amparo-Santos L, Soares MD. Alimentação escolar e constituição de identidades dos escolares: da merenda para pobres ao direito à alimentação. Cad Saúde Pública 2018; 34(4):e00142617.

19. Brasil. Nota Técnica no 02/2012 - COTAN/CGPAE/ DIRAE/FNDE. Regulamentação de cantinas escolares em escolas públicas do Brasil. Brasília: MEC; 2012.

20. Gabriel CG, Santos MV, Vasconcelos FAG, Milanez GHG, Hulse SB. Cantinas escolares de Florianópolis: existência e produtos comercializados após a instituição da Lei de Regulamentação. Rev Nutr 2010; 23(2):191-199.

21. Brasil. Portaria Interministerial $\mathrm{n}^{\circ} 1010$, de 08 de maio de 2006. Institui as diretrizes para a Promoção da Alimentação Saudável nas Escolas de educação infantil, fundamental e nível médio das redes públicas e privadas, em âmbito nacional. Diário Oficial da União 2006; 08 mai.

22. Brasil. Lei $n^{\circ} 06$, de 08 de maio de 2020. Dispõe sobre o atendimento da alimentação escolar aos alunos da educação básica no âmbito do Programa Nacional de Alimentação Escolar - PNAE. Diário Oficial da União 2020; 08 mai.

23. Freitas MCS, Minayo MCS, Ramos LB, FGV, Santos LA, Souza EC, Santos AC, Mota SE, Paiva JB, Bernardelli TM, Demétrio F, Menezes I. Escola: lugar de estudar e de comer. Cien Saude Colet 2013; 18(4):979-985.

24. Oliveira SE, Lígia AS, Dantas SM. Interações entre práticas alimentares e identidades: ressignificando a escola pública e a alimentação escolar. Cad Saúde Pública 2019; 35(11):e00217918.

25. Pan American Health Organization (PAHO). Ultra-processed food and drink products in Latin America: Trends, impact on obesity, policy implications. Washington: PAHO; 2015.

26. Leme ACB, Philippi ST, Toassa EC. O que os adolescentes preferem: os alimentos da escola ou os alimentos competitivos? Saude Soc 2013; 22(2):456-467.

27. Valentim EA, Almeida CCB, Taconeli CA, Osório $\mathrm{MM}, \mathrm{Schmidt} \mathrm{ST}$. Fatores associados à adesão à alimentação escolar por adolescentes de escolas públicas estaduais de Colombo, Paraná, Brasil. Cad Saúde Pública 2017; 33(10):e00061016.

28. Organização Pan-Americana da Saúde (OPAS). Plano de Ação para Prevenção da Obesidade em Crianças e Adolescentes. Washington: OPAS; 2015.

29. Chaves OC, Velasquez-Melendez G, Costa DAS, Caiaffa WT. Soft drink consumption and body mass index in Brazilian adolescents: National Adolescent Student Health Survey. Rev Bras Epidemiol 2018; 21(Supl. 1):e180010.
30. Nogueira FAM, Sichieri R. Associação entre consumo de refrigerantes, sucos e leite, com o índice de massa corporal em escolares da rede pública de $\mathrm{Ni}$ terói, Rio de Janeiro, Brasil. Cad Saúde Pública 2009; 25(12):2715-2724.

31. Henriques P, Dias PC, Burlandy L. A regulamentação da propaganda de alimentos no Brasil: convergências e conflitos de interesses. Cad Saúde Pública 2014; 30(6):1219-1228.

32. Organização Pan-Americana da Saúde (OPAS). Organização Mundial da Saúde (OMS). Políticas y programas alimentarios para prevenir el sobrepeso y la obesidad: Lecciones aprendidas. Washington: OPAS/ OMS; 2018.

33. Bertol CE, Souza M. Transgressões e adolescência: individualismo, autonomia e representações identitárias. Psicol Cien Prof 2010; 30(4):824-839.

34. Brasil. Ministério da Saúde (MS). Guia alimentar para a população brasileira. 2a ed. Brasília: MS; 2014.

35. Brasil. Ministério do Desenvolvimento Social e Combate à Fome. Marco de referência de educação alimentar e nutricional para as políticas públicas. Brasília: MDS; 2012.

36. Kwon AR, Yoon YS, Min KP, Lee YK, Jeon JH. Eating alone and metabolic syndrome: A population-based Korean National Health and Nutrition Examination Survey 2013-2014. Obes Res Clin Pract 2018; 12(2):146-157.

37. Fischler C. Commensality, society and culture. Soc Sci Information 2011; 50(3-4):528-548.

38. Dallacker M, Hertwig R, Mata J. Quality matters: A meta-analysis on components of healthy family meals. Health Psychol 2019; 38(12):1137-1149.

39. Guerra LDS, Espinosa MM, Bezerra ACD, Guimarães LV, Lima-Lopes MA. Insegurança alimentar em domicílios com adolescentes da Amazônia Legal Brasileira: prevalência e fatores associados. Cad Saúde Pública 2013; 29(2):335-348.

40. Cunha DT, Gonçalves HVB, Lima AFA, Martins PA, Rosso VV, Stedefeldt E. Regional food dishes in the Brazilian National School Food Program: Acceptability and nutritional composition. Rev Nutr 2014; 27(4):423-434.

Artigo apresentado em 14/02/2020

Aprovado em 27/07/2020

Versão final apresentada em 29/07/2020

Editores chefes: Romeu Gomes, Antônio Augusto Moura da Silva 